(92018 IEEE. Personal use of this material is permitted. Permission from IEEE must be obtained for all other uses, in any current or future media, including reprinting/republishing this material for advertising or promotional purposes, creating new collective works, for resale or redistribution to servers or lists, or reuse of any copyrighted component of this work in other works. This is the author's version of an article that has been published in the conference proceedings. The final version of record is available at https://doi.org/10.1109/PLANS.2018.8373509 


\title{
Relative Train Localization with Magnetic Field Measurements
}

\author{
Benjamin Siebler, Oliver Heirich and Stephan Sand \\ German Aerospace Center (DLR) \\ Institute of Communications and Navigation \\ Wessling, Germany \\ Email: benjamin.siebler@dlr.de
}

\begin{abstract}
In this paper a new method for relative train localization in global navigation satellite system (GNSS) denied areas is proposed. The proposed localization system is long-term stable and based solely on magnetometer and odometer measurements. The system utilizes that the magnetic field shows strong and time persistent variations along a railway track. Two trains driving on the same track will observe the same magnetic field variations but with a certain shift. This shift depends on the relative position of the trains and their speed. By measuring the train speed with an odometer it becomes possible to estimate the relative position by comparing the magnetometer and odometer measurements of two trains. In this paper we use cross-correlation to obtain the relative position estimate from a batch of measurements. A subsequent Kalman filter is used to smooth the estimate and to incorporate prior knowledge of the train dynamics. We further derive the Cramer-Rao lower bound (CRLB) for the relative position estimate to investigate the theoretically achievable localization accuracy and to approximate the variance of the relative position estimate in the update step of the Kalman filter. In an evaluation the feasibility and accuracy of the approach is shown based on measurement data collected with a train driving in a rural area. The results indicate that with the proposed method the relative position can be estimated with sub-meter accuracy.
\end{abstract}

\section{INTRODUCTION}

The capacity and flexibility in current railway systems is limited by the large safety distances between consecutive trains. To lower the safety distances while guaranteeing a safe operation, a higher degree of automation must be introduced into rail traffic. A new technique that can enable higher flexibility and increase the capacity of current track networks is virtual coupling. Virtual coupling uses a distance control loop to automatically keep a predefined distance between trains. This lowers the safety distances to a few meters and replaces the mechanical coupler between different trains. The replacement of the mechanical coupler with a control loop allows trains to drive in a platoon that can split and merge while driving. For virtual coupling, the estimation of the relative position or respectively the distance between trains is crucial. This estimate must be available in all environments including tunnels, underground and urban areas. In these scenarios GNSS signals are completely blocked or at least strongly distorted. In this paper, we therefore propose a new method for relative train localization that uses solely magnetic field and train speed measurements. The method is based on the observation that ferromagnetic infrastructure elements in the railway environment introduce strong distortions into the

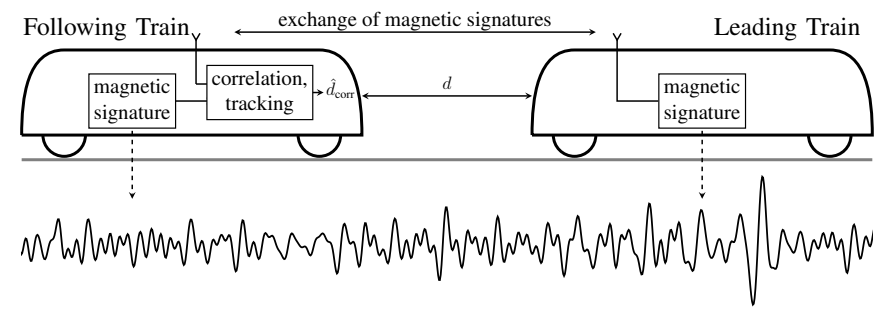

Fig. 1. Relative train localization with magnetic signatures.

earth magnetic field. Typically these infrastructure elements like rails, railroad ties and poles, have fixed positions that result in position dependent magnetic distortions. Therefore the distortions can be seen as a magnetic signature characteristic for a specific part of the track network. In our prior work, we investigated this magnetic signatures in a typical railway environment [1], [2]. We also showed a longterm stable absolute localization approach based solely on magnetometer and inertial measurement unit (IMU) measurements [3]. The idea of absolute localization with the magnetic field is also investigated for indoor environments and roads. In [4] and [5] the authors propose to use a particle filter to track the position of a pedestrian with measurements of the prior mapped magnetic field and measurements of an IMU. A similar approach for a wheeled indoor robot is introduced in [6]. Instead of an IMU, wheel speed sensors are incorporated to measure the displacement between consecutive measurements. In [7] the authors utilize a particle filter to localize a car on the road solely with the magnetic field. Like the other approaches mentioned before this requires a prior recorded map of the magnetic field. In this paper the focus is on estimating the relative position or distance between trains rather than the absolute position. More precisely, the so called on-track distance is estimated that accounts for the track geometry. The on-track distance is defined by the length of the track between two consecutive trains and therefore can be used directly as an input to the control loop of virtually coupled trains. In contrast to estimating the absolute position, the proposed relative localization approach does not require a prior recorded map of the magnetic field. Instead each train of a virtually coupled platoon creates a magnetic signature by measuring the magnetic field and relative position 
information from an odometer for a couple of hundred meters of track. To enable relative localization the trains share their signatures via a communication link with other trains in the platoon. The platoon drives on the same track and therefore the trains measure the same magnetic field and generate similar signatures. The only difference between the signatures is that they are shifted relative to each other. Therefore, to estimate the on-track distance between two trains the shift between their magnetic signatures must be found. This can be achieved by calculating the cross-correlation of two signatures. The most likely on-track distance is where the correlation function has its maximum value. A sketch of the basic idea is shown also in Fig. 1 with an exemplary magnetic field observed in the railway environment.

To suppress outliers and to filter the on-track distance estimate from the correlator, the correlator estimate is used as a measurement in a Kalman filter. The Kalman filter includes a model for the dynamics of the on-track distance and the relative train speed. Further the CRLB for the distance estimation is derived to investigate the theoretical localization accuracy that can be achieved with the magnetic signatures. The CRLB is then used as a quality measure for the correlator distance estimate. This is particularly important in the update step of the Kalman filter to set the measurement covariance to an appropriate value.

\section{MethodS}

\section{A. Magnetic field signatures for localization}

For the creation of the signatures the magnetic field is measured with a magnetometer and the train speed is recorded with a suitable sensor. By integrating the speed, it is possible to obtain the magnetic field with respect to the driven distance. Depending on the train speed the distance between two magnetic field samples is changing. To get an equally spaced signature the magnetic field is interpolated on an equidistant grid with a fixed spacing $\Delta s$. We call this process also spatial transformation since the magnetic field samples in the time domain are transformed into a magnetic signature that contains the measured magnetic field along the track which can be seen to be in the spatial domain. An example of a $200 \mathrm{~m}$ long signature is shown in Fig. 2. Due to the interpolation on a constant grid, the signature of train $i$ can be represented by a simple finite sequence

$$
\left(m_{n}^{i}\right)_{n=0, \ldots, N-1}
$$

In this sequence each value $m_{n}^{i}$ is the measured magnetic field at position $n \Delta s$. The position is relative to the current position of the train, for $n=100$ and $\Delta s=0.1 \mathrm{~m}$ this means that $m_{100}$ is the magnetic field $10 \mathrm{~m}$ behind the current train position and $m_{0}$ is the newest magnetometer measurement.

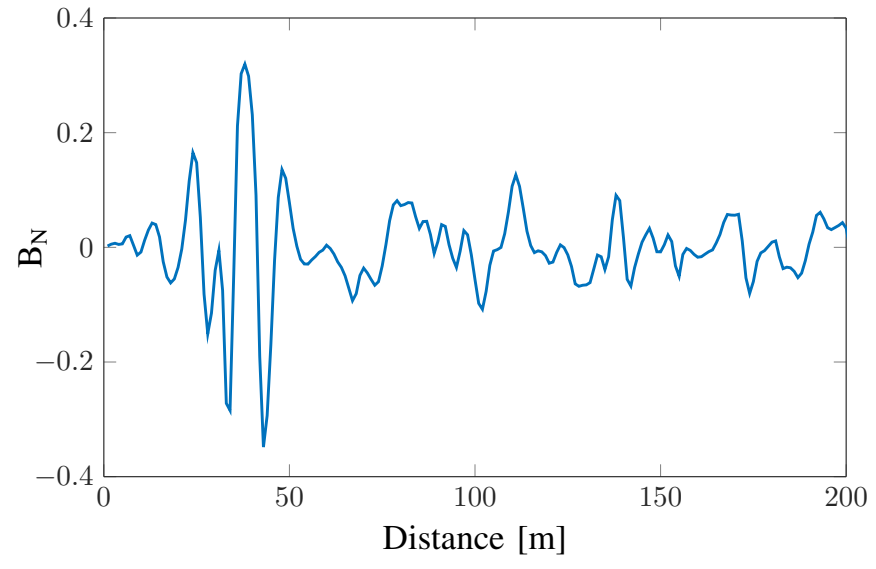

Fig. 2. Example of a $200 \mathrm{~m}$ long magnetic signature. $\mathrm{B}_{\mathrm{N}}$ is the measured magnetic field normalized with respect to the undistorted earth magnetic field.

\section{B. Relative position estimation}

The estimation of the on-track distance $d$ between train $i$ and train $j$ is based on the cross-correlation of their magnetic signatures

$$
\hat{d}_{\mathrm{corr}}=\Delta s \underset{m \in[0, N-1]}{\arg \max }\left(\sum_{n=0}^{N-m-1} m_{n+m}^{i} \cdot m_{n}^{j}\right) .
$$

When the distance between the trains is zero both signatures would contain the same magnetic field and the correlation function maximum should be located at shift $m=0$. Assuming that one of the signatures is perfectly recorded without noise, the cross-correlation is a matched filter and the maximum likelihood estimator of $d$. If the distance $d$ is greater than zero, the signatures contain parts that are common for both trains and parts that are exclusive to each signature. For a distance of $10 \mathrm{~m}$ and a signature length of $100 \mathrm{~m}$ the signature contain $90 \mathrm{~m}$ common magnetic field and $10 \mathrm{~m}$ that is exclusive to one signature. Even though this is not perfect for finding the shift between the two signatures and therefore the on-track distance, it still should be possible as long as the signatures contain a sufficiently large part of common magnetic field. For large distances this implies that also large signature lengths are required.

\section{Filtering of the cross-correlation estimate}

Errors in the measurements of the train speed and the magnetic field introduce errors in the estimate of the on-track distance (2). To filter this estimate, a Kalman filter with a time discrete kinematic model capturing the relative dynamics of two trains is used. The relative movement of the trains is modeled by a piecewise constant white acceleration model as proposed e.g. in [8]

$$
\mathbf{x}_{k}=\left[\begin{array}{c}
d_{k} \\
\dot{d}_{k}
\end{array}\right]=\left[\begin{array}{cc}
1 & T \\
0 & 1
\end{array}\right]\left[\begin{array}{c}
d_{k-1} \\
\dot{d}_{k-1}
\end{array}\right]+\left[\begin{array}{c}
\frac{1}{2} T^{2} \\
T
\end{array}\right] n_{\mathrm{Acc}}
$$

In (3) $T$ is the sampling period, $k$ the discrete time index and $\mathbf{x}=\left[\begin{array}{ll}d & \dot{d}\end{array}\right]^{T}$ is the state vector containing the on-track 
distance $d$ and the relative speed $\dot{d}$. The dynamics of the constant acceleration model is driven by the Gaussian process noise $n_{\text {Acc }} \sim \mathcal{N}\left(0, \sigma_{\text {Acc }}^{2}\right)$. The choice of $\sigma_{\text {Acc }}^{2}$ is important and should fit to the application. According to [8], the value should be in the range $0.5 \mathrm{a}_{\max } \leq \sigma_{\mathrm{Acc}} \leq a_{\max }$, where $a_{\max }$ is the maximal acceleration. For a train $a_{\max }$ is approximately $\pm 1 \mathrm{~m} / \mathrm{s}^{2}$, for the change of the relative speed this means that in the extreme case were the leading train is doing an emergency brake and the following train is fully accelerating $a_{\max }$ is $\approx 2 \mathrm{~m} / \mathrm{s}^{2}$.

The Kalman filter is updated with the on-track distance estimate $\hat{d}_{\text {corr }}$ from the cross-correlation, hence the measurement model is

$$
\hat{d}_{\text {corr }}=\mathbf{H} \mathbf{x}+w_{d}=\left[\begin{array}{ll}
1 & 0
\end{array}\right] \mathbf{x}+w_{d}
$$

with the measurement matrix $\mathbf{H}$ and the Gaussian measurement noise $w_{d} \sim \mathcal{N}\left(0, \sigma_{d}^{2}\right)$. The proper choice of the measurement noise variance $\sigma_{d}^{2}$ is discussed in the next section.

\section{Theoretical accuracy of the on-track distance estimate}

The quality of the on-track distance estimate depends on the shape of the magnetic field. For the Kalman filter update step it is desirable to adapt the variance of the measurement according to the actually measured magnetic field. To obtain a measure for this variance we propose to use the CRLB In particular we propose to use the CRLB for time delay estimation. In time delay estimation it is assumed that a known signal is transmitted to a receiver, were it is received with a certain time delay and noise. The estimation of this delay is identical to the problem of finding the shift between two magnetic signatures.

1) CRLB for time delay estimation: The CRLB for time delay estimation of a signal with additive white Gaussian noise is well known and can be found in the literature e.g. [9]

$$
\operatorname{Var}(\tau) \geq \frac{\sigma^{2}}{\sum_{k=0}^{N-1}\left[\frac{\partial}{\partial \tau} s(k T-\tau)\right]^{2}}
$$

Here $\sigma^{2}$ is the variance of the white Gaussian noise at the receiver that is superimposed on the transmitted signal $s(\cdot), \tau$ is the time delay and $T$ is the sampling period at the receiver. For the purpose in this paper this formulation cannot be applied directly because the signal $s(\cdot)$, here the magnetic signature, is not known analytically. Instead only a measurement sequence is known. A transformation of the CRLB (5) into the frequency domain according to [9] solves this issue

$$
\operatorname{Var}(\tau) \geq \frac{1}{\frac{E_{S}}{N_{0} / 2} \beta}=\frac{\sigma^{2} T}{E_{S} \beta}
$$

where $\beta$ is the mean square bandwidth

$$
\beta=\frac{\int_{-\infty}^{+\infty}(2 \pi f)^{2}|S(f)|^{2} d f}{\int_{-\infty}^{+\infty}|S(f)|^{2} d f}
$$
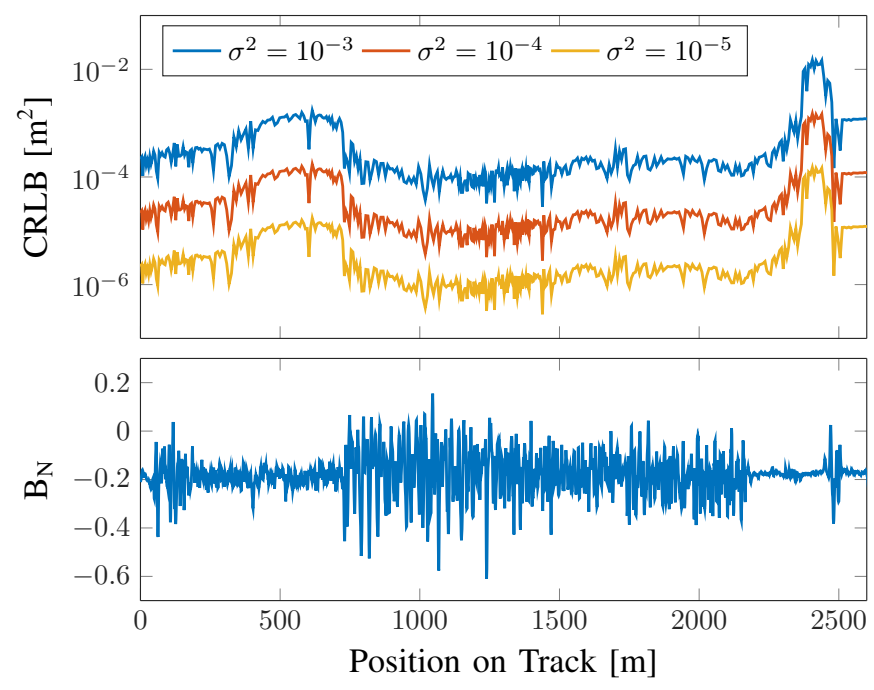

Fig. 3. Magnetic field along a $2.6 \mathrm{~km}$ long track and the corresponding CRLB of the on-track distance for different values of the magnetometer noise variance.

and $E_{s}$ is the signal energy

$$
E_{S}=\int_{0}^{T_{\mathrm{obs}}} s(t)^{2} d t
$$

For discrete sequences the integrals in (7) and $(8)$ have to be approximated. For $\beta$ we can write

$$
\beta \approx \frac{4 \pi^{2} \sum_{n=0}^{N / 2} f_{n}^{2}\left|S_{n}\right|^{2}}{\sum_{n=0}^{N / 2}\left|S_{n}\right|^{2}}
$$

where $S_{n}$ are the coefficients of the fast Fourier transform (FFT) of the signal $s(\cdot)$ and $f_{n}=\frac{1}{N \Delta T} n$ are the corresponding discrete frequencies. The signal energy is obtained from the Riemann sum approximation of (8)

$$
E_{S}=T \sum_{k=0}^{N-1}|s(k T)|^{2} .
$$

2) CRLB for on-track distance estimation: For on-track distance estimation the CRLB is equal to (6) when the time delay $\tau$ becomes the on-track distance $d$ and the sampling period $T$ is replaced with the distance $\Delta s$ between two samples in the magnetic signature. The mean square bandwidth (9) and the signal energy (10) are now calculated based on the sequence $(m)_{k}$ and $\sigma$ becomes the standard deviation of the magnetometer sensor noise.

Fig. 3 shows the CRLB for a $2.6 \mathrm{~km}$ long track for different values of $\sigma^{2}$. The CRLB is calculated with the latest $200 \mathrm{~m}$ of magnetic field. For the CRLB at track position $500 \mathrm{~m}$ therefore the magnetic field recorded from 300 to $500 \mathrm{~m}$ is considered. To reduce the effect of measurement noise on the CRLB the magnetic field was filtered in the spatial domain with a Butterworth filter with a cut-off frequency of $0.5 \mathrm{~m}^{-1}$ before 
the CRLB was calculated. The magnetometer we used during our measurements was low-cost and had a noise variance of $\sigma^{2} \approx 3 \cdot 10^{-5}$. This means that from a theoretical point of view the achievable variance of the on-track distance estimate is in the range of $1 \cdot 10^{-6} \mathrm{~m}^{2}$. This low value is caused by the high signal energy in the magnetic field that can be seen also from the example in Fig.2. In combination with the small variance of the sensor noise a large signal to noise ratio (SNR) can be observed.

3) Variance for the Kalman filter update step: It is clear that in a realistic scenario the CRLB will be never attained by an estimator because of different error sources that are not considered in the CRLB. The first error source is the spatial transformation due to erroneous train speed measurements. A second error source is that the two signatures are measured with different sensors at different positions. Temporary disturbances in the magnetic field caused e.g. by changing electromagnetic fields or by passing trains might affect only one of the sensors. Further, when the on-track distance is not zero a part of the compared magnetic signatures will contain measurements from different parts of the track. The variance and the error of the on-track distance estimate are therefore expected to be significantly higher than proposed by the CRLB. To account for this errors and to get an estimate for the variance of the correlator estimate $\hat{d}_{\text {corr }}$ the CRLB in (6) is scaled and $\sigma^{2}$ is replaced with a constant $c$ that has to be chosen empirically

$$
\operatorname{Var}\left(\hat{d}_{\text {corr }}\right) \approx \frac{c \Delta s}{E_{S} \beta} .
$$

Intuitively 11 is plausible since one can expect that the variance of $\hat{d}_{\text {corr }}$ decreases for signatures with a high signal energy and mean square bandwidth.

\section{E. Outlier rejection}

Besides normal errors that should be accounted for by $\operatorname{Var}\left(\hat{d}_{\text {corr }}\right)$, we also observed outliers in the correlator distance estimate. A simple outlier rejection scheme based on the Mahalanobis distance prevents updating the Kalman filter with outliers. A measurement is rejected when the Mahalanobis distance of the Kalman filter innovation

$$
d_{\text {Maha }}=\sqrt{\left(d_{\text {corr }}-\mathbf{H x}\right)^{T} \mathbf{S}^{-1}\left(d_{\text {corr }}-\mathbf{H x}\right)}
$$

is above an empirically defined constant threshold. In (12) $\mathrm{S}$ is the innovation covariance matrix from the Kalman filter that depends on the state and the measurement covariance.

\section{Evaluation}

The measurements for the evaluation were recorded in spring 2014 on a rural railway track network in northern Germany. In the evaluation two hours of data collected on $\approx 60 \mathrm{~km}$ of tracks is used. The tracks are mainly located in suburban, rural and forest environments. The train was powered by a diesel engine and the maximum speed during the measurements was $50 \mathrm{~km} / \mathrm{h}$. Throughout the evaluation the signature length was set to $200 \mathrm{~m}$ and the sample distance was $\Delta s=0.1 \mathrm{~m}$.

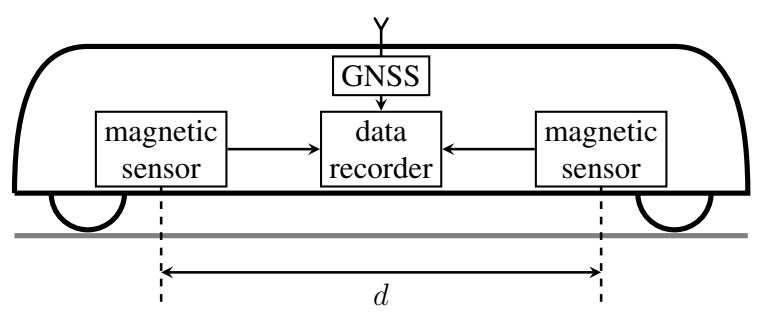

(a)

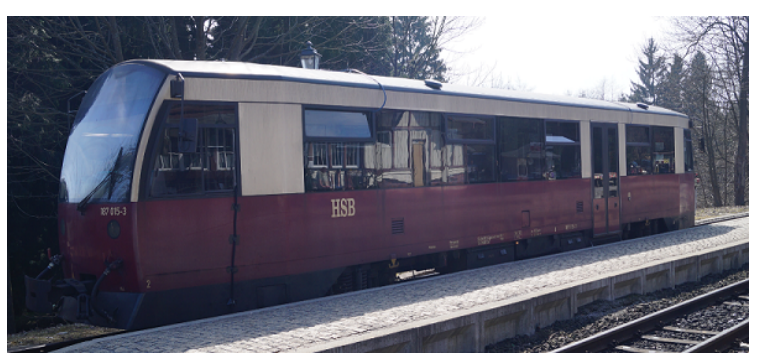

(b)

Fig. 4. (a) Measurement setup: The two magnetometers were fixed to the floor inside the train cabin and the speed was measured with a GNSS receiver. (b) Picture of the diesel train from the Harzer Schmalspurbahnen that was used during the measurements.

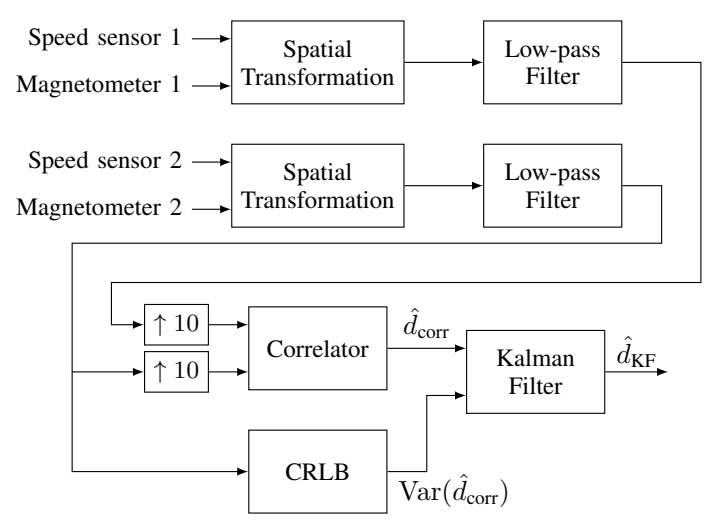

Fig. 5. Block diagram of relative train localization system.

\section{A. Measurement setup}

The setup for the measurements is depicted in Fig.4(a), To emulate two trains driving with an constant distance two magnetometer were mounted on the floor of the diesel train, shown in Fig.4(b), with a distance of $9.59 \mathrm{~m}$. The magnetometers are low-cost sensors that are integrated in an Xsens MTi-G-700 IMU. For the speed measurements the train was equipped with a single frequency LEA-M8T GNSS receiver from u-blox that was also used to synchronize the measurements of the magnetometers. The speed and navigation solution is calculated from the receiver raw data with an extended Kalman filter. The measurement rates were $100 \mathrm{~Hz}$ for the magnetometers and $1 \mathrm{~Hz}$ for the GNSS receiver. For the magnetic signatures the measurements of the downwards facing magnetometer axis is used. The Kalman filter is updated with a distance estimate from the cross-correlation with $10 \mathrm{~Hz}$. In the evaluation the 
spatial transformation relies on the speed of the GNSS receiver but in principle also other speed sensors can be used. Typical speed sensors for trains are e.g. wheel turn sensors, ground facing Doppler radar and optical sensors. Here GNSS is used because typically it is not possible to connect to the train sensors or it is not allowed due to safety concerns.

\section{B. Odometer error simulation}

In the evaluation first the same GNSS speed is used to perform the spatial transformation of booth magnetometer measurement sequences. Due to the constant sensor distance a slowly varying bias on the GNSS speed introduces the same errors in the spatial transformation of both signatures. This is done to minimize the effect of unknown speed errors on the results. In a second step then the impact of speed errors on the accuracy of the on-track distance estimate is evaluated by artificially adding two different types of errors on the GNSS speed measurements.

1) Zero mean noise: The first simulated error is white Gaussian noise. To find an appropriate value for the noise standard deviation, the value of the Doppler radar DRS 051S1 from DEUTA is used. The radar was especially developed for railway applications and has according to the datasheet [10] a standard deviation of $0.11 \mathrm{~m} / \mathrm{s}$. The distribution of the errors is not stated but for simplicity a Gaussian distribution is used in the simulation.

2) Constant bias: A speed bias error can be caused e.g. by wheel slip when a wheel turn sensor is used. Typically slip can be observed while the train is accelerating and decelerating. The bias error is simulated by adding a constant value to the speed measurements.

\section{Magnetic signature preconditioning}

After the spatial transformation, the signatures contain errors due to the magnetometer measurement noise and speed errors. To remove high frequency errors, we apply a Butterworth low-pass filter with a cut-off frequency of $0.5 \mathrm{~m}^{-1}$ since the localization relevant information in the magnetic field is allocated at low frequencies. One of this filtered signatures is used to calculate the variance of $\hat{d}_{\text {corr }}$ required in the Kalman filter update step. For the correlation the filtered signatures are additionally up-sampled by a factor of ten to get a distance between two signature values of $1 \mathrm{~cm}$. This increases the resolution of the correlator output and reduces the discretization effects. Fig.5 shows the block diagram of the overall system architecture.

\section{RESULTS}

The performance of the proposed system is evaluated in terms of the root mean square error (RMSE) of the on-track distance estimate $\hat{d}$

$$
\operatorname{RMSE}=\sqrt{\frac{1}{N} \sum_{n=1}^{N}\left(\hat{d}_{n}-d\right)^{2}}=\sqrt{\frac{1}{N} \sum_{n=1}^{N} \epsilon_{n}{ }^{2}}
$$

with the distance error $\epsilon$. In Table $\llbracket$ the RMSE obtained from a $2 \mathrm{~h}$ long data set recorded on over $60 \mathrm{~km}$ of tracks is shown.
TABLE I

RMSE, $99 \%$ QUANTILE $q_{99}$ AND MAXIMUM MAGNITUDE $\epsilon_{\text {MAX }}$ OF THE DISTANCE ERROR.

\begin{tabular}{lccrcrr}
\hline & \multicolumn{3}{c}{ GNSS speed } & \multicolumn{3}{c}{ Noisy speed } \\
Algorithm & RMSE [m] & $q_{99}[\mathrm{~m}]$ & $\epsilon_{\max }[\mathrm{m}]$ & RMSE [m] & $q_{99}[\mathrm{~m}]$ & $\epsilon_{\max }[\mathrm{m}]$ \\
\hline KF $+\sigma_{\mathrm{c}}$ & 0.27 & 0.52 & 7.19 & 0.29 & 0.55 & 7.49 \\
KF+CRLB & 0.20 & 0.52 & 3.96 & 0.22 & 0.56 & 4.26 \\
Cross-correlation & 3.76 & 0.57 & 168.08 & 3.75 & 0.58 & 168.03 \\
\hline
\end{tabular}

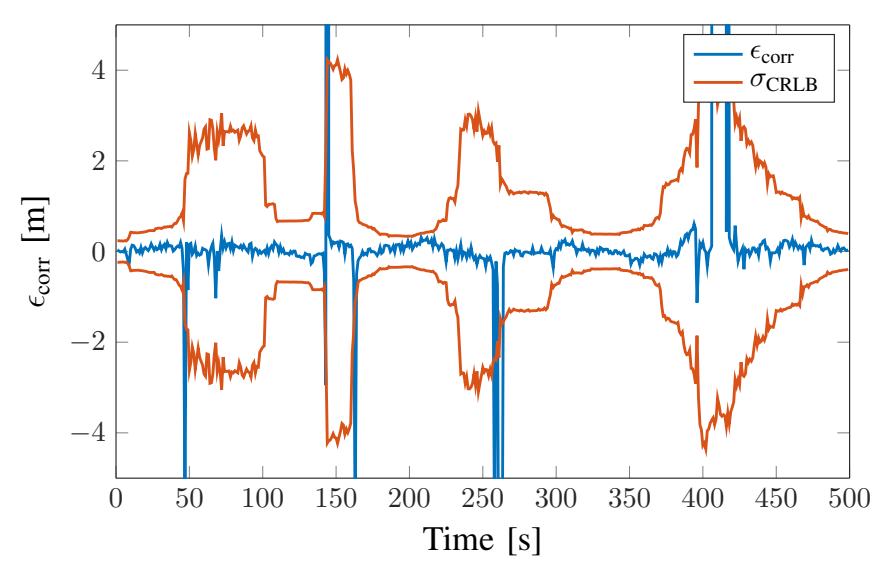

Fig. 6. Standard deviation $\sigma_{\mathrm{CRLB}}$ derived from the CRLB in comparison to the unfiltered correlator estimation error.

Additionally the table includes the $99 \%$ quantile $q_{99}$ and the maximum amplitude $\epsilon_{\max }$ of the on-track distance estimation error. The first three columns are the results where we used for both signatures the same GNSS speed in the spatial transformation. In contrast to this, the last three columns are the results for the case where the signatures are generated with additive noise on the speed measurements. The noise is different for each signature and was simulated as described before. In Table I the distance estimate from the correlation is compared to two Kalman filter implementations. The two implementations differ only in respect to the measurement variance in the update step. The first implementation uses a constant variance of $\sigma_{c}^{2}=0.09 \mathrm{~m}^{2}$ that was chosen to ensure that the estimation errors are within the estimated state covariance. The second implementation uses (11) with a scaling constant of $c=0.3$ to calculate the variance $\sigma_{\mathrm{CRLB}}^{2}$ based on the current signature. In Fig. 6 an example of $\sigma_{\text {CRLB }}^{2}$ calculated for a $500 \mathrm{~s}$ long measurement sequence is shown in comparison to the correlator estimation error. It can be seen that $\sigma_{\mathrm{CRLB}}$ actually follows the shape of the correlator errors. It is not a perfect match but here it must be considered that only one realization of the correlator output is compared to a variance. For a better comparison multiple runs on the same track would be required. Then the variance of the correlator error could be compared to $\sigma_{\text {CRLB }}^{2}$. A comparison of the RMSE values in Table 1 shows that small noise on the speed has almost no effect on the distance estimation. Further it can be seen that using a Kalman filter reduces the RMSE significantly as one would expect. The gain is caused by the outlier rejection in combination with the kinematic model in 


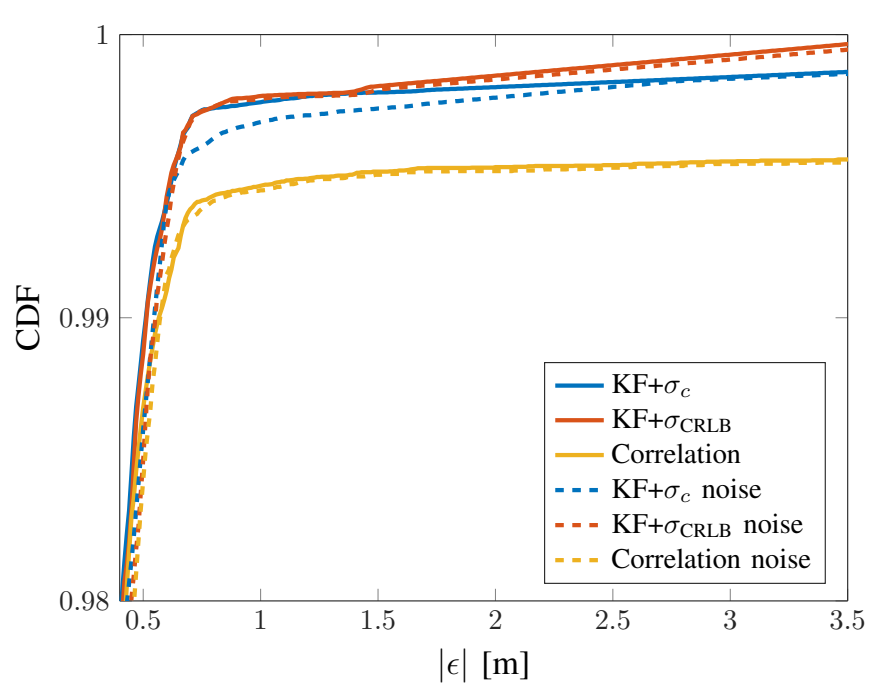

Fig. 7. CDF of the distance estimation error for the different algorithms.

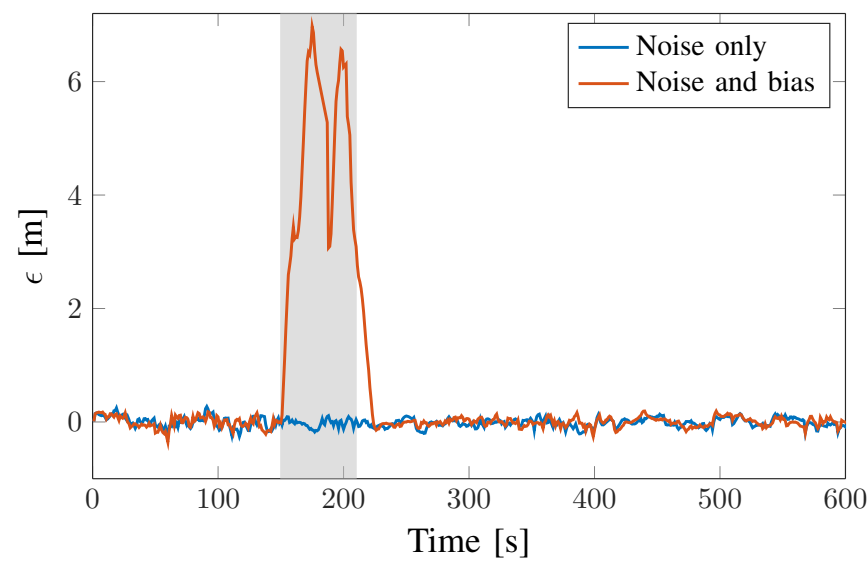

Fig. 8. On-track distance error in the presence of a $0.5 \mathrm{~m} / \mathrm{s}$ speed bias between 151 to $211 \mathrm{~s}$ (indicated by the grey area).

(3). The improvement of the Kalman filter is also clearly visible in the cumulative distribution function (CDF) of $|\epsilon|$ in Fig.77. The results for the two Kalman filter implementations show that using $\sigma_{\mathrm{CRLB}}$ instead of a constant variance improves the estimation accuracy in terms of the RMSE and seems to improves the outlier suppression. In respect to the $99 \%$ quantile a difference between the two Kalman filter implementations is not noticeable. For the cross-correlation the $q_{99}$ value is also close to the Kalman filer values, showing that the RMSE of the cross-correlation is mainly increased due to the outliers.

In Fig. 8 we see the result of a bias error on the speed measurements. Between 151 to $211 \mathrm{~s}$ a bias of $0.5 \mathrm{~m} / \mathrm{s}$ was added to the noisy speed measurements used to generate one of the signatures. This introduces an linearly growing error in the spatial transformation up to $30 \mathrm{~m}$. Fig. 8 shows the severe influence of a speed bias on the distance estimation. It is therefore important that the speed measurements are unbiased or the bias is estimated.

\section{CONCLUSION}

The results show the feasibility of the proposed method for relative train localization. Overall an RMSE below $0.3 \mathrm{~m}$ was achieved. Noise on the speed measurements only has a small impact on the attainable accuracy. In contrast to this, a bias error on the speed measurements introduces large errors into the spatial transformation and the distance estimate. For accurate relative localization it is therefore crucial to have speed sensors with a small bias. The evaluation further showed that even though the variance derived from the CRLB was not matching the correlator error perfectly incorporating it into a Kalman filter still was beneficial in comparison to the naive approach with a constant variance. By comparing the evaluation results with the theoretical results from the CRLB, it can be concluded that the quality of the magnetometers is not a limiting factor. The accuracy of the relative localization mainly depends on the quality of the speed measurements used in the spatial transformation and the shape of the magnetic field along the track.

\section{ACKNOWLEDGMENT}

Thanks goes to the Harzer Schmalspurbahnen and their staff for providing the rolling stock for the measurements.

\section{REFERENCES}

[1] O. Heirich, B. Siebler, and E. Hedberg, "Study of train-side passive magnetic measurements with applications to train localization," Journal of Sensors, Juni 2017.

[2] O. Heirich and B. Siebler, "Train-side passive magnetic measurements," in 2015 IEEE International Instrumentation and Measurement Technology Conference (I2MTC) Proceedings, May 2015, pp. 687-692.

[3] B. Siebler, O. Heirich, and S. Sand, "Bounding ins positioning errors with magnetic-field-signatures in railway environments," in Proceedings of the 30th International Technical Meeting of The Satellite Division of the Institute of Navigation (ION GNSS+ 2017), September 2017, pp $3224-3230$.

[4] M. Frassl, M. Angermann, M. Lichtenstern, P. Robertson, B. J. Julian, and M. Doniec, "Magnetic maps of indoor environments for precise localization of legged and non-legged locomotion," in 2013 IEEE/RSJ International Conference on Intelligent Robots and Systems, Nov. 2013, pp. 913-920.

[5] J. Haverinen and A. Kemppainen, "Global indoor self-localization based on the ambient magnetic field," Robotics and Autonomous Systems, vol. 57, no. 10, pp. 1028-1035, Oct. 2009

[6] H.-S. Kim, W. Seo, and K.-R. Baek, "Indoor Positioning System Using Magnetic Field Map Navigation and an Encoder System," Sensors, vol. 17, no. 3, p. 651, Mar. 2017.

[7] J. A. Shockley and J. F. Raquet, "Navigation of ground vehicles using magnetic field variations," Navigation, vol. 61, no. 4, pp. 237-252, 2014, navi.70.

[8] Y. Bar-Shalom and X.-R. Li, Estimation and Tracking: Principles, Techniques, and Software. Artech House, 1993.

[9] S. M. Kay, Fundamentals of statistical signal processing. Prentice Hall PTR, 1993.

[10] DEUTA Sensors, DEUTA-WERKE, May 2017. 